\title{
Integration of Systematic Screening for Tuberculosis in Outpatient Departments of Urban Primary Health-Care Facilities in Zambia: A Case Study of Kitwe District
}

\section{Davy Wadula Zulu}

Department of Health Policy and Management, School of Public Health, University of Zambia

Adam Silumbwe ( $\sim$ adam.silumbwe@umu.se)

Department of Epidemiology and Global Health, Umeå University

\section{Patricia Maritim}

Department of Health Policy and Management, School of Public Health, University of Zambia Joseph Mumba Zulu

Department of Health Policy and Management, School of Public Health, University of Zambia

\section{Research Article}

Keywords: facilities, implementation, integration, primary healthcare, systematic screening, tuberculosis

Posted Date: January 5th, 2022

DOI: https://doi.org/10.21203/rs.3.rs-998743/v2

License: (c) (1) This work is licensed under a Creative Commons Attribution 4.0 International License.

Read Full License 


\section{Abstract \\ Background}

Tuberculosis (TB) is the leading cause of death from a single infectious agent globally, yet 3 million cases are missed every year. The World Health Organization recommends systematic screening of suspected active TB patients among those visiting the healthcare facilities. While many countries have scaled-up systematic screening of TB, there has been limited assessment of the extent of its integration into the health system. This study sought to explore factors that shaped the integration of systematic screening of TB in outpatient departments of primary healthcare facilities in Kitwe district, Zambia.

\section{Methods}

This was a qualitative case study with health providers including district managers, TB focal point persons and laboratory personnel working in six purposively selected primary healthcare facilities. Data was collected through key informant $(n=8)$ and in-depth $(n=15)$ interviews. Data analysis was conducted using QDA Miner software and guided by Atun's Integration framework.

\section{Results}

Integration was facilitated by perceptions of the magnitude of the TB burden, alignment of the intervention with national TB aspirations, knowledge of stakeholder interests, power and values, regular performance management and intra-facility collaboration. Constraining factors included external partners' influence in the TB screening program, unbalanced incentivization mechanisms, donor-driven financing and social determinants of health such as gender and stigma including the COVID-19 pandemic.

\section{Conclusion}

Systematic screening of TB is not well integrated into the primary healthcare facilities to capture all those suspected with active TB that make contact with the health system. Finding the missing TB cases will, therefore, require contextual adaptation of the systematic screening for TB and strengthening the health system.

\section{Contributions To Literature}

- The incomplete integration of systematic screening for tuberculosis might partially explain the low TB notification rates in LMIC 
- The factors that shape integration of systematic screening for TB are applicable to similar low resource settings and therefore require the attention of TB program managers to enhance implementation success

- The findings can be used to improve the integration of similar TB interventions into health systems in similar settings.

\section{Background}

Globally, tuberculosis (TB) remains one of the top 10 causes and leading cause of death from a single infectious agent, killing about 1.5 million people each year $(1,2)$. It is estimated that $85 \%-95 \%$ of these deaths could be prevented with early detection, diagnosis and appropriate treatment. Yet almost 3 million TB cases are missed (either not diagnosed or diagnosed but not notified) (1). Thus, the importance of promptly identifying TB cases and linking them to care cannot be over emphasized.

In the past 25 years, global TB control has seen the adoption of three main strategies. The Direct Observed Treatment (DOTS) strategy of 1994 to 2005 emphasized passive case finding (3). This was followed by the STOP TB strategy of 2006 to 2015 which emphasized intensified case finding in health facilities, communities and congregate settings (4). Currently, the END TB strategy since 2016 proposes active case finding and contact tracing among populations at risk (5). Through these strategies, 58 million TB deaths were averted between the years 2000 and 2018 (6). However, there are fundamental gaps hampering the success of these global strategies, particularly in resource-constrained settings(7).

Evidence suggests that the missed TB cases are not really missing as most of them are actively engaging with the health system which is failing to appropriately capture them (8). This is due to various factors including low TB screening and testing capacity, poor understanding of the screening protocols, inadequate knowledge by the health providers to suspect TB, low diagnostic capacities and shortages of inputs(10-12). Further, the growing interest in community-based active case finding (ACF) strategies especially in resource-constrained settings has shifted the attention from $(13,14)$ facility-based intensified TB case finding even though it is more cost-effective and efficient $(15,16)$.

The World Health Organization (WHO) underscores missed TB cases as a significant contributor to continued high prevalence and incidence of TB in low- and middle-income countries (LMIC) and recommends systematic screening of all suspected active TB patients who come to the health facility (6). Systematic screening for TB is the systematic identification of people with suspected active TB, in a predetermined target group, using tests, examinations or other rapid procedures (17). While many LMICs have scaled-up systematic screening of TB, there has been limited assessment of the extent of its integration into the health system(1).

Zambia is one of the top 30 high TB burden countries with an estimated incidence of $346 / 100,000$ population compared to a global average of 130/100,000 (19). Disease control efforts in Zambia are guided by the National Strategic Plan for tuberculosis prevention, care and control 2017-2021 (NSP) and 
the national TB manual of 2017. Both policy documents outline systematic screening for TB at health facility level as a key strategy for detection of TB cases $(20,21)$. This is done through symptomatic screening for cough, blood in sputum, chest pains, fever, night sweats and weight loss (Figure 1).

Despite the implementation and roll out of systematic screening for TB over the past 10 years in Zambia, about $50 \%$ of symptomatic TB patients who seek healthcare from various health facilities are not investigated for TB (22). This situation might explain the stagnant TB notification rates of less than $60 \%$ since $2015(2,23)$.

Integration of systematic screening for TB into the health system greatly contributes to optimal treatment outcomes (24). However, few studies have been done in Zambia to understand how systematic screening for TB is integrated into primary healthcare systems. This study focuses on the integration of the systematic screening for TB in the outpatient departments (OPDs) of primary health facilities with a view to understand contextual factors that contribute to missing TB cases at the health facilities. Specifically, this paper reports on the perceptions and experiences of healthcare workers ( $\mathrm{HCWs)} \mathrm{on} \mathrm{the} \mathrm{integration} \mathrm{of}$ the systematic screening for TB in the OPDs and factors that shape this integration. We define integration as the extent, pattern, and rate of adoption and eventual assimilation of systematic screening for TB into each of the critical functions of a health system (25).

\section{Methods}

\section{Research design}

A qualitative case study design was employed as it is appropriate for exploring integration in the context of an urban primary healthcare facilities of Kitwe district (26). This design allowed us to uncover context specific implementation issues affecting integration of systematic screening in primary healthcare facilities. We used the consolidated criteria for reporting qualitative research (COREQ) to guide the research process (additional file).

\section{Study setting}

Kitwe district is located in the central part of the Copperbelt Province of Zambia as shown in Figure 2 below. It is the second most populated district in Zambia after the capital, Lusaka with about 66 percent of the population below the age of 25 years (27). The main economic activities in the district are mining, agriculture and tourism. At the time of study, the district delivered its healthcare services through 3 hospitals and 39 primary healthcare facilities coordinated by the District Health Office. All 39 primary health facilities screened for TB but only 12 had TB diagnostic capabilities and the rest referred sputum samples or patients to these diagnostic health facilities. Kitwe district was chosen as a case because it had high TB incidence and the high number of TB patients that had interacted with health facilities but were not investigated for TB (22).

\section{Participant recruitment and sampling approach}


All primary health care facilities in Kitwe District were listed and six were purposively selected with maximum variation in TB notifications (three with highest and three with lowest TB notifications) with guidance from the District Health Management Team (DHMT). The final selection of health facilities was subject to informed consent from each health facility in-charges. District TB managers were selected purposively by the research team and contacted through the help of the facility in-charges who in turn helped in the identification of OPD clinicians, laboratory personnel (where available), and health facility TB focal point persons. District TB managers were chosen because they are the ones with oversight of the TB programs including the systematic screening for TB while the HCWs were selected because they are the ones who are the implementers of systematic screening for TB within health facilities. The sample size was determined by the principle of saturation (28)

\section{Data collection}

Data were collected between November 2020 and January 2021 through a total of twenty-three $(\mathrm{N}=23)$ face-to-face Key Informant (KIIs) and In-Depth interviews (IDIs) (Table 1). Interview guides developed according to the objectives of the study and the theoretical framework were used for data collection. All interviews were conducted in English and lasted between forty-five and eighty minutes. The interviews were digitally recorded with consent from participants and field notes were taken. The KIls for the district TB managers were conducted at the District Health Office and all the other interviews were conducted at the respective health facilities observing privacy and COVID-19 prevention measures. Data were recorded using a digital recorder and transcribed verbatim. During and at the end of the interviews, a summary of the responses was repeated to each participant to confirm if their responses were captured and interpreted correctly.

\section{Data analysis}

A thematic analysis approach to explore relationships and patterns in qualitative data was used (29). The data analysis process started during data collection by DWZ in order to learn and refine the interview questions. It was an on-going process that involved arranging the field notes according to themes in relation to the objectives and the framework. DWZ listened to participants' recorded responses several times for familiarization and were transcribed verbatim. Field notes and comments were regularly reviewed in line with the themes and a list of major ideas that surfaced were added to a code list which was imported into QDA Miner software. Six transcripts were shared with AS, PM and JMZ to verify the content and consolidate the coding. Subsequent iterative discussions among the research team allowed for additional modifications of the coding structure. Both inductive and deductive coding approaches were used to ensure both framework-guided and emergent subthemes in the data were captured. Data was triangulated across different categories of participants and health facilities.

The data collection, analysis and reporting of findings was guided by Atun's Integration Framework which posits that the integration of health interventions into health systems is influenced by; the nature of the problem being addressed, the intervention, the adoption system, the health system characteristics, and the broader context(30). From this, we theorized that the nature of the TB problem such as its burden 
may influence actors' perspectives towards TB guidelines thus shaping the integration process. Attributes of the systematic screening for TB such as the complexity and compatibility of its components with the work culture and values of the actors in the adopting system-including program managers and health workers and with the health system characteristics such as resources, capacity building, demand creation, performance and regulatory systems may also influence the integration process. Factors in the broader context such as the demographic, economic, political and socio-cultural factors may shape integration of the systematic screening for TB into the health system by affecting any of the above factors (Figure 3 ).

\section{Ethical approval and consent to participate}

Ethical clearance was sought from the University of Zambia Biomedical Research Ethics Committee (REF. 1015-2020). Permission was also sought from the National Health Research Authority, the Ministry of Health as well as Kitwe District authorities to conduct the study. Signed informed consent was obtained from all participants. COVID-19 preventive measures were adhered to through provision of face masks, hand sanitizer and social distancing during data collection. All the participants were de-identified to guarantee confidentiality. All methods were carried out in accordance with relevant guidelines and regulations.

\section{Results}

We present our findings on the factors that shape the integration of systematic screening into the OPDs of primary healthcare facilities. The themes have been organized around the constructs of Atun's framework (30) on integration of health interventions into health systems, namely: characteristics of the problem, attributes of the intervention, the adoption system, the health system characteristics and the broader context (Table 2). Although data were collected from various participant categories, no major differences in the discussions were noted and views specific to a particular participant category are noted within the manuscript.

\section{Factors that shape the integration of systematic screening for Tuberculosis in OPDs of primary healthcare facilities}

\section{Nature of the TB problem}

\section{Perception of the TB burden}

It was interesting to find differing perceptions of the burden of TB among HCWs in the same healthcare facilities. Most clinicians perceived the TB burden to be high and considered TB to be a serious public health problem both at district and health facility level due to the high numbers of TB patients seen in the OPDs. The surrounding environment, mostly low social-economic housing from which these patients 
originated also played a role in driving TB. Some facility in-charges thought that the burden was lessening because of the effective community TB screening programs that had been implemented in the past. This perception of a high TB burden increased the index of suspicion for TB and facilitated integration as stated by a clinician:

"Looking at ... the environment of this community and the number of [TB] cases that I see, I would say the burden is high and so I make sure I do my best...to catch them." Clinician-02, LP

The facility in-charge from the same facility argued that;

"As for TB, I can say not so much. Why? Because we don't have a lot of TB patients due to various community screening programs conducted in the past." IC-02, LP

\section{The intervention}

\section{Alignment with HCWs' training, skills and work schedules}

Most HCWs agreed that the principles and concepts of the systematic screening for TB were relatively easy to understand and apply. They were within their in-service training and skills, making it easy for them to adopt and apply it. The compatibility of systematic screening for TB with clinicians' and laboratory staff's routine work schedule facilitated the integration process as they did not have to change the flow of patients.

"...our training is enough. Our work is not disturbed... We just need to keep in mind that we need to ask about TB in all patients who come through." Clinician-06, LP

However, the intervention was not compatible in most cases with the pre-service training and work schedules of the appointed facility TB focal point persons who were all nurses. They had to adapt by learning completely new concepts such as TB drugs and their side effects, the treatment algorithms, follow-up of patients, reporting systems and templates through in-service trainings. Those who could not adapt well were thus unable to integrate the intervention into the OPDs. Moreover, some of them were still attached to and working in their routine departments thus having a divided work schedule which hindered full implementation and integration of systematic screening for TB into the OPD.

"I am a nurse but now I need to work like a clinical officer, I had to learn the drugs and how to treat a TB patient... through mentorship. I also write all the TB reports for the facility. I never thought I could change like this..."TB-FPP-01, HP

\section{Complexity in screening of children}

All clinicians noted that it was difficult to integrate systematic screening for children in the OPDs because children presented with atypical symptoms which were not included in the screening tools. In addition, 
children who suspected cases were unable to expectorate sputum and it was time-consuming and difficult to perform a gastric lavage. The additional time required to conduct the systematic screening for TB effectively created pressure of work, especially in high volume healthcare facilities, which was a barrier to integration.

"With the adults, it's a straightforward thing, but with the children, there are hiccups because they are presenting not with all the features of TB on the screening tools and lavage is time-consuming, difficult and uncomfortable." Clinician-05, HP.

\section{The adopting system}

\section{Stakeholders' interests, values and power}

Stakeholders' knowledge of each other's interests, values and power facilitated integration as stakeholders adopted activities and strategies in a bid to fulfill the interest of others which sustained the consistent implementation of the systematic screening for TB in the OPDs. For instance, the district team's knowledge of the funders' financial power and interest to achieve project targets and improve the district TB indicators encouraged them to ensure HCWs sustain TB screening and notification.

"Our partners want results... we are making sure that all our TB programs are done and the facilities are doing what they are supposed to do as far as TB screening and notifications are concerned." Manager-01

Similarly, HCWs aligned their activities to the district and partners' values and interests to meet the TB targets set for them in order to receive more funding and recognition. By so doing they increased the extent of assimilation of the systematic screening into the OPDs while meeting the community expectations of satisfactory TB services.

"Someone [a CBV] goes out ... will collect any sputum... just to meet the quarterly target and get paid but that defeats science. So, we put more incentives for a positive TB result... for them to do it right always." Lab-02, HP

\section{Incentivization mechanisms}

Most OPD clinicians noted that there was an imbalance in incentivization structures among the implementers of the intervention because the TB focal point persons in the health facilities enjoyed more incentives through workshops and trainings. The TB focal point persons would receive more recognition from the district team and funders for targets achieved than the clinicians. This incentivization mechanisms created differences which demotivated clinicians from routinely screening for TB and hindered sustainability of integration.

"...you see, these guys, the focal persons when they see that this workshop involves money, they will go, yet they don't screen. Instead of sending the actual people who screen the patients. ... It is very 
demotivating..."Clinician-05, HP

Further, HCWs knowledge of the financial incentives provided to the CBVs in relation to meeting targets enabled the them to allocate more incentives to positive TB results than absolute numbers screened, thus maintaining quality through fidelity and encouraging integration.

\section{External partners influence}

Most facility level participants sited the ever-increasing influence of external partners in what activities were implemented when and who could be involved as a hinderance to the integration of systematic screening for TB in their health facilities. HCWs' lack of involvement at the planning stage coupled with prioritization of funders' activities over their own made them question the ownership of the TB screening program and the legitimacy of the TB screening targets given to them by the district and cooperating partners. The dependence on donor-funding for most of the TB programs including training of HCWs and TB screening activities seemed to validate their concerns of ownership. This compromised the acceptability, adoption and fidelity of the systematic screening for TB and its integration into the routine OPD services as it would be done only when it was closely supervised.

"Sometimes we have programs but partners come in with other programs... They even come with targets that we do not know where they came from..." IC-03, LP

\section{Characteristics of the health system}

\section{Consistent and decentralized performance management}

$\mathrm{HCW}$ s agreed that regular monitoring and supervision by the district team motivated them to consistently screen for TB in the OPD. Decentralizing this performance management to the health facilities through facility in-charges and heads of departments within the primary healthcare facilities was effective in ensuring fidelity and sustainability. The decentralized supervision further fostered the adoption of systematic screening for TB into all entry points of the health facility. Regular data reviews at facility level enabled quick adaptive measures and effective integration of TB screening into the OPDs.

"Before the district come... I am the center of the supervision for screening; but in each department, we have heads. So, with the help of those heads in those departments, we are the ones who are doing it... every head of the Department has been given the responsibility to ensure that such activities [TB screening] are going on every day." TB-FPP-1, HP.

\section{Continuous capacity building}

Continuous capacity building through on-site mentorship of HCWs maintained high index of suspicion for TB and ensured compatibility of the intervention and fidelity at all times with less TB patients being missed. The competences built in the HCWs made them more confident to accept and adopt the 
systematic screening for TB into their routine work. Onsite mentorship within the facility was considered to be a good initiative for building capacity and fostered integration because it involved all HCWs and brought up contextualized solutions to challenges faced by HCWs in the integration process.

"...So, the onsite membership, for me, it has really helped us. ... it makes me confident to do it [integrate systematic screening for TB into routine work]. They [the district mentorship team] keep on coming for mentorship and technical support... to maintain high suspicion index for TB..."IC-01, HP

\section{Integrated outreach TB screening services}

All participants noted that combining mobile TB screening services in the community with under- 5 clinics and other periodic programs like child health week increased its acceptance in the health facilities which facilitated integration. Some TB focal point persons and facility in-charges further added that the use of CBVs and the involvement of traditional healers, who are trusted members of society, in referring suspected TB patients were found to be good ways of increasing demand and acceptability of the systematic screening for TB.

"So, when you use community members [CBVs] in outreach TB screening and education while doing other programs like under-5 clinics and child health week...It will be very easy for them to accept even when they come to the facility." IC-03, HP

\section{Negative health worker attitudes}

Facility in-charges lamented over the lack of interest and poor attitude towards systematic screening for TB among some HCWs in the OPDs who only screened when there was someone monitoring them. This lack of interest in TB screening by the HCWs compromised their acceptance, adoption and consistent application of the intervention and thus was a barrier to its integration int the OPDs. The lack of interest bred a negative attitude towards patients making it hard for patients to accept TB screening.

"Some have no interest in screening for TB...because we may know how to screen quite alright but ...if we don't care how we treat them [patients]... can they accept it? ...I think the main reason is just the attitude." IC-04, LP

\section{The Broader Context}

\section{Political will}

District managers were encouraged by the political will from the government to ensure that all facilities had infrastructure, HCWs, commodities and equipment to provide TB screening services despite financial constraints. They stated that the provision of these supplies in the facilities would increase the integration of systematic screening for TB into the OPDs because the testing would be quicker and of good quality. 
"... when you look at equipment and staff, I think we have been given. I think we have more than fifteen or so laboratories which are actually doing screening using the GeneXpert for instance, it has helped us to do tests faster and I think with quality... We may need more but I am saying... I think the government is doing quite well." Manager-01

\section{Socio-cultural misconceptions and gender norms}

Most participants confirmed the persistent presence and negative effects of socio-cultural misconceptions despite the sensitizations on TB. Beliefs that TB was contracted by sleeping with a woman during their menses or by being bewitched prompted patients to seek help from traditional healers and reduced the acceptability of the systematic screening. The association of TB to HIV also propagated stigma which hindered people from screening for TB for fear of societal rejection and humiliation. Some TB focal point persons and facility in-charges noted that women were more affected by TB-associated stigma because society placed the "burden of marriage" on them. Most women would refuse to test for TB due to the possible consequences they would face from their partners such as shame, beatings and even divorce if they were found with it.

"...So even when you find them with TB, they wouldn't want to disclose to the partners because they are ... scared that those partners may chase them away or divorce, or things like that. ... some would ...totally refuse to test." IC-05, HP

\section{The COVID-19 pandemic}

All participants agreed that during the peak period of COVID-19 in the first and second quarters of 2020, OPD attendances, health education sessions and duration of clinical assessment reduced for fear of contracting the disease. The guideline not to wear masks while processing TB samples conflicted with the COVID guidelines to were masks at all times making laboratory personnel reluctant to process TB samples. Further, there was a shift in clinician concentration to COVID which compromised the integration of systematic screening for TB into the OPDs.

"... COVID has caused a lot of challenges ... the concentration has slightly shifted... from these other causes... Instead ... you tend to screen faster so that the patient leaves your room \{laughing\}." Clinician-05, HP

\section{Discussion}

This study explored factors that shape the integration of systematic screening for TB into the OPDs of primary healthcare facilities in order to improve the detection, notification and treatment. Facilitators of integration included perceived high burden of TB, alignment of systematic screening for TB with HCWs' skills, training and work schedules, stakeholders' knowledge of each other's interests, power and values, consistent performance management, continuous capacity building, and integrated outreach TB screening services in a broader context of political will. Barriers to integration included complexity in 
screening of children, unbalanced incentivization mechanisms, legitimacy of the systematic screening for TB, negative staff attitudes, socio-cultural misconceptions and gender norms and the COVID-19 pandemic.

HCWs' perception of a high burden of TB in their respective catchment areas was associated with a high suspicion index for TB which facilitated integration of systematic screening for TB into the OPDs. There is evidence that TB cases are missed due to lack of clinical suspicion (31-33) and that a high index of suspicion is associated with higher TB screening and detection rates $(34,35)$. Given the importance of perception of the problem on the index of suspicion, a deliberate effort must therefore be made by policy makers to engage frontline HCWs to raise awareness of the magnitude of TB for successful, integrated and sustained implementation.

Unsurprisingly, the pressure of work and difficulty to screen for TB in children discouraged HCWs from integrating the systematic screening for TB into the OPDs. This is confirmed by other studies which found that HCWs were reluctant to screen for TB in children due to children's nonspecific clinical presentation, their inability to expectorate sputum and the difficulty of aspirating a sputum sample (36-42). Apart from hindering integration, this systematic leaving out of children might create a health system propagated inequity in implementation which could be mitigated by increasing the numbers and capacities of HCWs. However, faster and easy integration of the systematic screening for TB can take place if the intervention is aligned with the HCWs skills, training and work schedules. Similarly, a systematic review of staffreported barriers and facilitators to the implementation processes of hospital-based interventions showed that suitability of an intervention to HCWs influenced how easily it was integrated(43).

Within the adopting system, similar to other studies (44-47) stakeholders' knowledge of each other's interests and values enhanced collaborations and by-in which facilitated integration. However, the unintended effects of incentives such as crowding-out intrinsic motivation and deterioration in service quality and quantity following their withdrawal (48) coupled with HCWs lack of ownership of the TB screening program driven by the unwelcome external partners influence, questionable source and legitimacy of the screening targets and donor-driven funding demotivated HCWs thus constrained integration. We therefore reiterate that policy makers engage all stakeholders at all stages of the intervention outlining the purpose of the intervention, the context in which it will be applied, the roles of each stakeholder and the funds available before they decide to incentivize it in order to encourage by-in and sustainability.

Consistent and decentralized performance management and continuous capacity building of HCWs through regular on-site mentorships enabled quick contextualized solutions to the integration process. The preference of on-site mentorship by HCWs (49) implies that contextualized, provider-tailored on-site mentorship should be prioritized by national TB programs and various stakeholders to accelerate and sustain integration. Dependance of these activities on donor funding, however, undermined local ownership and sustainability similar to studies in Nigeria (50) thus constraining the integration. Negative attitudes and behavior of the HCWs towards TB screening constrained integration as it significantly 
correlates to detection of new TB cases (51). Unfortunately, most TB programs, including systematic screening, do not consider behavioral factors as important for their adoption and sustainability.

The important role played by political will in the integration of interventions in health systems as part of the broader context is supported by studies in Community-based Healthcare workers, chronic illnesses and cervical cancer programs (52-55). Stigma perpetuated by the association of TB to HIV discouraged patients, especially women, from being tested for TB for fear of being victims of societal discrimination and rejection similar to a national-wide survey on TB-associated stigma in Ethiopia(56). This study, therefore, agrees with Miller et. al (2017) that all interventions designed to increase case detection must address stigma and its interaction with gender (57) for inclusive integration. The fear of and the shift of clinical concentration to COVID-19 compromised quality and increased likelihood of missing TB cases (58). Initial sensitization on emergencies such as COVID-19, therefore, must be targeted at HCWs to allay fears and maintain integrated services.

\section{Study limitations and strengths}

One limitation of the study is that the sample from Kitwe District may not represent views of other primary health facilities in Zambia, but still provides critical information and learning points on the integration of systematic screening for TB into the primary health system. In addition, the perceptions of HCWs and their supervisors are only one part of the picture, and their views needed to be complemented

with views of community members. However, the rich description of phenomena, our triangulation of data and adherence to principles of trustworthiness all helped in developing a credible and dependable account that we believe provides a valuable contribution to the knowledge base on factors that shape the integration of systematic screening of TB into the primary health system and could be transferable to similar settings.

\section{Conclusion}

Integration of systematic screening for TB into the primary healthcare system is not very complicated for HCWs and can improve indicators in TB detection, treatment and notification. Efforts to facilitate this integration in Zambia have been made through aligning the intervention to HCWs skills, training and work schedules, stakeholder engagement, decentralized and continuous performance management and political will over the years. However, integration still remains a challenge due to factors around the TB problem itself, the intervention to be integrated, the adopting system, characteristics of the health system and the broader context. These include the low index of suspicion for TB, complexity in screening children, unequal incentives among HCWs, questionable local ownership of the intervention, lack of interest and poor attitudes of HCWs towards integration, socio-cultural misconceptions about TB and shocks such as the COVID-19 pandemic.

Finding the missing TB cases will, therefore, require contextual adaptation of the systematic screening for TB and strengthening the health system if the intervention is to significantly contribute to meeting the 
2025 and 2035 local and global TB targets. Further research to quantify the extent of integration of systematic screening for TB in each of the health system building blocks is required and could be combined with findings from this study to design interventions targeted to strengthen specific weak aspects of integration.

\section{Abbreviations}

ACF......... Active Case Finding

CHWs......... Community Health Workers

DOTS.........Direct Observed Treatment, Short-course

HBCs.........High TB burden countries

HCWs.......... Healthcare workers

LMIC......... Low- and Middle-Income Countries

$\mathrm{MoH} . . . . . . . . .$. Ministry of Health in Zambia

NSP.............National Strategic Plan for Tuberculosis Prevention, Care and Control 2017-2021

OPDs.......... Outpatient Departments

TB............Tuberculosis

WHO.........World Health Organization

\section{Declarations}

\section{Ethical approval and consent to participate}

Ethical clearance was sought from the University of Zambia Biomedical Research Ethics Committee (REF. 1015-2020). Permission was also sought from the National Health Research Authority, the Ministry of Health as well as Kitwe District authorities to conduct the study. Signed informed consent was obtained from all participants. COVID-19 preventive measures were adhered to through provision of face masks, hand sanitizer and social distancing during data collection. All the participants were de-identified to guarantee confidentiality. All methods were carried out in accordance with relevant guidelines and regulations.

\section{Consent for publication}

Not applicable 
Availability of data and materials

The datasets used and/or analyzed during the current study are available from the corresponding author on reasonable request.

\section{Competing interests}

The authors declare that they have no competing interests.

\section{Funding statement}

DWZ is a recipient of a TDR scholarship under the Postgraduate Training Scheme in Implementation Research at the University of Zambia. We are grateful for the financial support for the training scheme provided by UNICEF, UNDP, World Bank and the WHO Special Program for Research and Training in Tropical Diseases (TDR). The funders were not involved in any part of the study.

\section{Author contributions}

DWZ conceived the research question and designed the study. DWZ, AS, PM and JMZ oversaw investigation and research methodology. DWZ was responsible for data acquisition, curation and analysis. JMZ oversaw project administration. DWZ, PM and AS wrote the original draft of this manuscript. All authors reviewed and edited the manuscript and approved the final version.

\section{Acknowledgements}

We appreciate the efforts, commitment, and contributions of the Kitwe District Health Management Team in coordinating and facilitating data collection. Sincere thanks go to all healthcare providers for accepting to be part of this study and for sparing time from their busy schedules to attend the interviews.

\section{References}

1. World Health Organization. Global Tuberculosis Report 2020. Geneva: World Health Organization; 2020.

2. World Health Organization. Global tuberculosis report 2019. WHO publications. Geneva; 2019.

3. World Health Organization. An Expanded DOTS Framework for Effective Tuberculosis Control. Geneva; 2002.

4. World Health Organization. WHO Policy on TB Infection Control in Health-Care Facilities, Congregate Settings and Households. Geneva; 2009.

5. World Health Organization. Implementing the End TB strategy: The Essentials. Geneva; 2015.

6. World Health Organization. People-centred framework for tuberculosis programme planning and prioritization-user guide. Geneva; 2019.

7. World Health Organization. WHO Global TB Programme: End TB Accelerator Package. Geneva; 2019. 
8. Padayatchi N, Daftary A, Naidu N, Naidoo K, Pai M. Tuberculosis: Treatment failure, or failure to treat? Lessons from India and South Africa. BMJ Glob Heal. 2019 Jan 1;4(1):1097.

9. Kweza PF, Van Schalkwyk C, Abraham N, Uys M, Claassens MM, Medina-Marino A. Estimating the magnitude of pulmonary tuberculosis patients missed by primary health care clinics in South Africa. Int J Tuberc Lung Dis. 2018;22(3):264-72.

10. Kwan A, Daniels B, Saria V, Satyanarayana S, Subbaraman R, McDowell A, et al. Variations in the quality of tuberculosis care in urban India: a cross-sectional, standardized patient study in two cities. PLoS Med. 2018;15(9):e1002653.

11. Ohene S-A, Bonsu F, Hanson-Nortey NN, Toonstra A, Sackey A, Lonnroth K, et al. Provider initiated tuberculosis case finding in outpatient departments of health care facilities in Ghana: yield by screening strategy and target group. BMC Infect Dis. 2017;17(1):1-11.

12. Subbaraman R, Nathavitharana RR, Satyanarayana S, Pai M, Thomas BE, Chadha VK, et al. The tuberculosis cascade of care in India's public sector: a systematic review and meta-analysis. PLoS Med. 2016;13(10):e1002149.

13. Biermann O, Lönnroth K, Caws M, Viney K. Factors influencing active tuberculosis case-finding policy development and implementation: a scoping review. bmjopen.bmj.com. 2019 Dec 11;9(12).

14. Kerrigan D, West N, Tudor C, Hanrahan CF, Lebina L, Msandiwa R, et al. Improving active case finding for tuberculosis in South Africa: informing innovative implementation approaches in the context of the Kharitode trial through formative research. Heal Res policy Syst. 2017;15(1):1-8.

15. Hanrahan CF, Nonyane BAS, Mmolawa L, West NS, Siwelana T, Lebina L, et al. Contact tracing versus facility-based screening for active TB case finding in rural South Africa: A pragmatic clusterrandomized trial (Kharitode TB). PLoS Med. 2019;16(4).

16. Bigogo G, Cain K, Nyole D, Masyongo G, Auko JA, Wamola N, et al. Tuberculosis case finding using population-based disease surveillance platforms in urban and rural Kenya. BMC Infect Dis. 2018;18(1):1-12.

17. World Health Organization. Systematic screening for active tuberculosis: an operational guide. World Health Organization; 2015.

18. Legido-Quigley H, Montgomery CM, Khan P, Atun R, Fakoya A, Getahun H, et al. Integrating tuberculosis and HIV services in low- and middle-income countries: a systematic review. Trop Med Int Heal. 2013 Feb 1;18(2):199-211.

19. World Health Organization. Country profiles For 30 high TB burden countries. Geneva; 2020.

20. Ministry of Health Z. The National Tuberculosis and Leprosy Control Program - TB Manual 2017. Lusaka: Government Printers; 2017.

21. Ministry of Health Z. Zambia National Strategic Plan for Tuberculosis 2017-2021 "Towards Elimination ". Lusaka; 2017.

22. Ministry of Health Z. Zambia National TB Prevalence Survey 2013-2014. Lusaka; 2015. 
23. World Health Organization. Global tuberculosis report 2015. 20th ed. World Health Organization; 2016. $192 \mathrm{p}$.

24. Shigayeva A, Atun R, McKee M, Coker R. Health systems, communicable diseases and integration. Health Policy Plan. 2010;25(suppl_1):i4-20.

25. Atun R, Menabde N. Health Systems and Systems Thinking. In Open University Press, McGraw Hill Education; 2008. p. 121-40.

26. Ritchie J, Lewis J, Nicholls CM, Ormston R. Qualitative research practice: A guide for social science students and researchers. sage; 2013.

27. Zambia Statistical Agency. Zambia National Census 2010. 2011.

28. Hagaman AK, Wutich A. How many interviews are enough to identify metathemes in multisited and cross-cultural research? Another perspective on Guest, Bunce, and Johnson's (2006) landmark study. Field methods. 2017;29(1):23-41.

29. Braun V, Clarke V. Using thematic analysis in psychology. Qual Res Psychol. 2006;3(2):77-101.

30. Atun R, De Jongh T, Secci F, Ohiri K, Adeyi O. Integration of targeted health interventions into health systems: A conceptual framework for analysis. Health Policy Plan. 2010;25(2):104-11.

31. Kiwuwa M, Karamagi C, Mayanja K. Patient and health service delay in pulmonary tuberculosis patients attending a referral hospital: a cross-sectional study. BMC Public Health. 2005;5.

32. Sendagire I, Loeff MS Van der, Mubiru M, Konde-Lule J, Cobelens F. Long Delays and Missed Opportunities in Diagnosing Smear-Positive Pulmonary Tuberculosis in Kampala, Uganda: A CrossSectional Study. PLoS One. 2010;5(12):e14459.

33. Assefa D, Belachew F, Wondimagegn G, Klinkenberg E. Missed pulmonary tuberculosis: a cross sectional study in the general medical inpatient wards of a large referral hospital in Ethiopia. BMC Infect Dis 2019 191. 2019 Jan 17;19(1):1-7.

34. Kagujje M, Chilukutu L, Somwe P, Mutale Id J, Chiyenu K, Lumpa M, et al. Active TB case finding in a high burden setting; comparison of community and facility-based strategies in Lusaka, Zambia. PLoS One. 2020;

35. Li J, Liu XQ, Jiang SW, Li X, Yu F, Wang Y, et al. Improving tuberculosis case detection in underdeveloped multi-ethnic regions with high disease burden: A case study of integrated control program in China. Infect Dis Poverty. 2017 Nov 29;6(1):1-9.

36. Marais BMP. Recent advances in the diagnosis of childhood tuberculosis. adc.bmj.com. 2011;

37. Nelson L, Schneider E, Wells C, Pediatrics MM-, 2004 U. Epidemiology of childhood tuberculosis in the United States, 1993-2001: the need for continued vigilance. Am Acad Pediatr. 2004;

38. Mulenga $\mathrm{H}$, Tameris $\mathrm{M}, \ldots \mathrm{KL}-\mathrm{TP}, 2015 \mathrm{U}$. The role of clinical symptoms in the diagnosis of intrathoracic tuberculosis in young children. ncbi.nlm.nih.gov. 2015;

39. Singh AR, Kumar A, Shewade HD, Dhingra B. Poor adherence to TB diagnosis guidelines among under-five children with severe acute malnutrition in central India: A missed window of opportunity? PLoS One. 2021 Mar 1;16(3 March):e0248192. 
40. Nelson \& Wells C. Global epidemiology of childhood tuberculosis. - Int J Tuberc. 2004;

41. Montenegro S, Gilman R, ... PS-Cl, 2003 U. Improved Detection of Mycobacterium tuberculosis in Peruvian Children by Use of a Heminested IS6110 Polymerase Chain Reaction Assay. academic.oup.com. 2003;

42. Nicol M\& ZH. New specimens and laboratory diagnostics for childhood pulmonary TB: progress and prospects. Paediatr Respir. 2011;

43. Geerligs L, Rankin NM, Shepherd HL, Butow P. Hospital-based interventions: a systematic review of staff-reported barriers and facilitators to implementation processes. Implement Sci 2018 131. 2018 Feb 23;13(1):1-17.

44. Lu AD, Kaul B, Reichert J, Kilbourne AM, Sarmiento KF, Whooley MA. Implementation Strategies for Frontline Healthcare Professionals: People, Process Mapping, and Problem Solving. J Gen Intern Med. 2021 Feb 1;36(2):506-10.

45. Leeman J, Birken SA, Powell BJ, Rohweder C, Shea CM. Beyond "implementation strategies": Classifying the full range of strategies used in implementation science and practice. Implement Sci. 2017 Nov 3;12(1):125.

46. Concannon TW, Meissner P, Grunbaum JA, McElwee N, Guise JM, Santa J, et al. A new taxonomy for stakeholder engagement in patient-centered outcomes research. Vol. 27, Journal of General Internal Medicine. 2012. p. 985-91.

47. Miech EJ, Rattray NA, Flanagan ME, Damschroder L, Schmid AA, Damush TM. Inside help: An integrative review of champions in healthcare-related implementation. SAGE Open Med. 2018 Jan 1;6:205031211877326.

48. Deci EL, Ryan RM. A Meta-Analytic Review of Experiments Examining the Effect of Extrinsic Rewards on Intrinsic Motivation. psycnet.apa.org. 1999;

49. Manabe YC, Zawedde-Muyanja S, Burnett SM, Mugabe F, Naikoba S, Coutinho A, et al. Rapid improvement in passive tuberculosis case detection and tuberculosis treatment outcomes after implementation of a bundled laboratory diagnostic and on-site training intervention targeting midlevel providers. Open Forum Infect Dis. 2015 Jan 1;2(1).

50. Ogbuabor DC, Onwujekwe OE. Governance of tuberculosis control programme in Nigeria. Vol. 8, Infectious Diseases of Poverty. BioMed Central Ltd.; 2019. p. 1-11.

51. Ratnasari NY, Marni M, Husna PH. Knowledge, Behavior, and Role of Health Cadres in The Early Detection of New Tuberculosis Case in Wonogiri. J Kesehat Masy. 2019 Dec 22;15(2):235-40.

52. Inouye J, Boland M, Nigg C, ... KS-H medical, 2011 undefined. A center for self-management of chronic illnesses in diverse groups. ncbi.nlm.nih.gov.

53. Mwanahamuntu MH, Sahasrabuddhe V V., Kapambwe S, Pfaendler KS, Chibwesha C, Mkumba G, et al. Advancing Cervical Cancer Prevention Initiatives in Resource-Constrained Settings: Insights from the Cervical Cancer Prevention Program in Zambia. PLOS Med. 2011 May;8(5):e1001032.

54. Moon TD, Silva-Matos C, Cordoso A, Baptista AJ, Sidat M, Vermund SH. Implementation of cervical cancer screening using visual inspection with acetic acid in rural Mozambique: successes and 
challenges using HIV care and treatment programme investments in Zambézia Province. J Int AIDS Soc. 2012 Apr 1;15(2):17406.

55. Zulu JM, Kinsman J, Michelo C, Hurtig A-K. Integrating national community-based health worker programmes into health systems: a systematic review identifying lessons learned from low-and middle-income countries. BMC Public Heal 2014 141. 2014 Sep 22;14(1):1-17.

56. Datiko DG, Jerene D, Suarez P. Stigma matters in ending tuberculosis: Nationwide survey of stigma in Ethiopia. Vol. 20, BMC Public Health. BioMed Central Ltd.; 2020. p. 1-10.

57. Miller C, Huston J, Samu L, Mfinanga S, Hopewell P, Fair E. "It makes the patient's spirit weaker":

Tuberculosis stigma and gender interaction in Dar es Salaam, Tanzania. Int J Tuberc Lung Dis. 2017 Nov 1;21:S42-8.

58. Buonsenso D, lodice F, Sorba Biala J, Goletti D. COVID-19 effects on tuberculosis care in Sierra Leone. Vol. 27, Pulmonology. Elsevier Espana S.L.U; 2021. p. 67-9.

\section{Tables}

Table 1: IDI and KII participants

\begin{tabular}{|ll|}
\hline Data collection method/participants & Number of interviews \\
\hline Key informant interviews & \\
\hline District TB program managers & 2 \\
\hline Health facility in-charges & 6 \\
\hline Total KIls & $\mathbf{8}$ \\
\hline In-depth interviews & \\
\hline Laboratory Personnel & 5 \\
\hline TB Focal Point Person & 4 \\
\hline OPD Clinician & 6 \\
\hline Total IDIs & $\mathbf{1 5}$ \\
\hline Total Interviews & $\mathbf{2 3}$ \\
\hline
\end{tabular}

Table 2: Key thematic categories 


\begin{tabular}{|c|c|}
\hline Broad themes & Sub-theme \\
\hline The TB burden/problem & - Perception of the TB burden \\
\hline \multirow[t]{2}{*}{ The intervention } & - Alignment with HCWs skills, training and work schedules \\
\hline & - Complexity in screening children \\
\hline \multirow[t]{3}{*}{ The adoption system } & - Stakeholders' knowledge of each other's interests, values and power \\
\hline & - Unequal Incentivization mechanisms \\
\hline & $\begin{array}{l}\text { - Doubted ownership and legitimacy of the systematic screening for } \\
\text { TB }\end{array}$ \\
\hline \multirow{4}{*}{$\begin{array}{l}\text { Health system } \\
\text { characteristics }\end{array}$} & - Consistent and decentralized performance management \\
\hline & - Continuous capacity building \\
\hline & - Integrated TB screening outreach services \\
\hline & - Staff interests and attitudes \\
\hline \multirow[t]{3}{*}{ Broad context of integration } & - Political will \\
\hline & - Socio-cultural misconceptions and gender norms \\
\hline & - The COVID-19 pandemic \\
\hline
\end{tabular}

\section{Figures}




\section{Triage}

Separate the patient from the queue

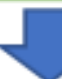

Referral

Refer the patient to the clinician as a priority case

Patient education

Counsel the patient on clinical presentation and diagnosis of pulmonary tuberculosis

Take infection prevention measures and provide the patient a surgical mask

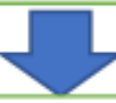

Clinical assessment

Conduct clinical assessment for TB and take vital signs, temperature, respiratory rate

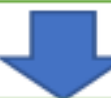

\section{Diagnosis and treatment}

After clinician's review, separate the patient in the presumed TB bay Collect sputum samples for examination

After confirming the diagnosis of TB, start TB treatment, notify (admit if patient's condition requires admission)

\section{Figure 1}

Phases of systematic screening for TB in out-patient departments 


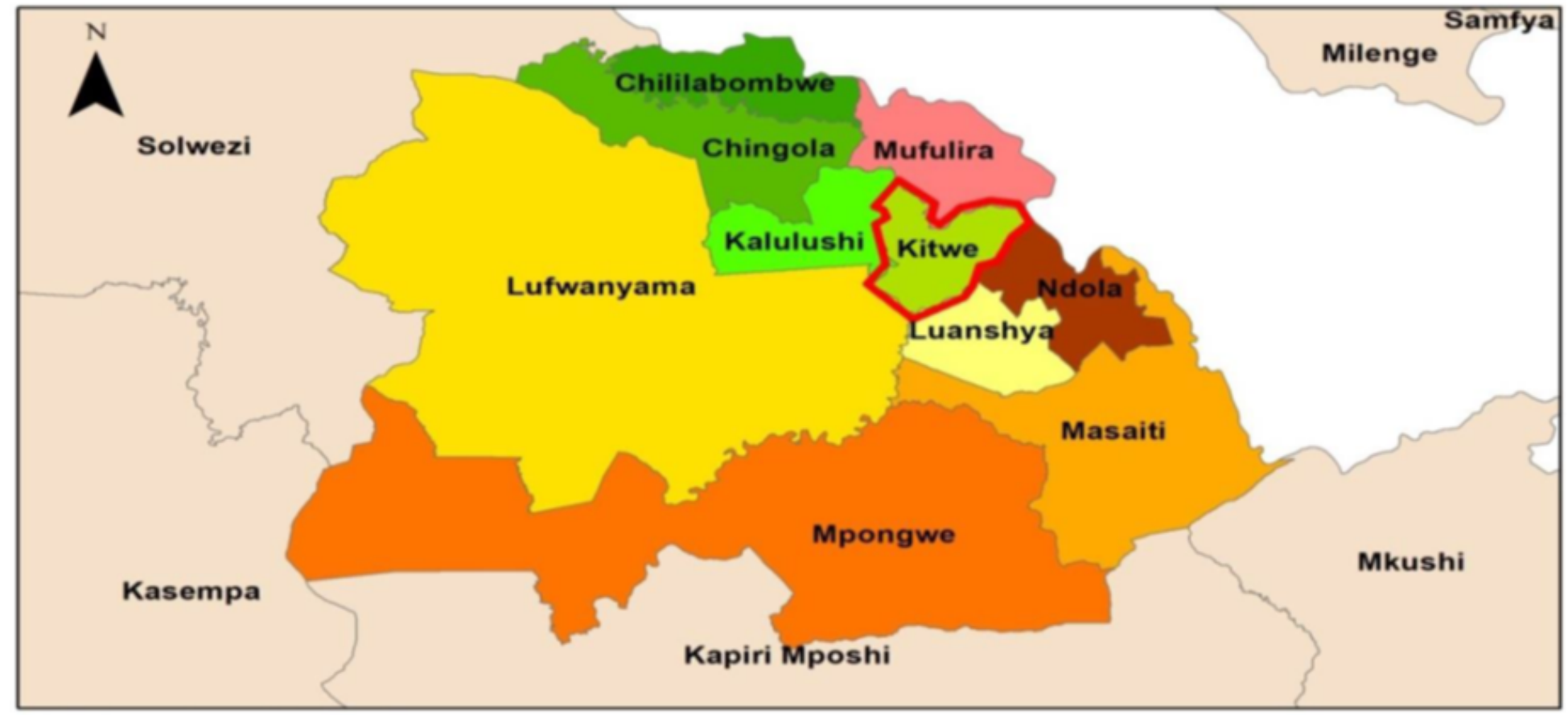

SCALE : 1: 2000000

Figure 2

Location map of Kitwe district

(Source: Provincial administrative office, Copperbelt Province, 2019)

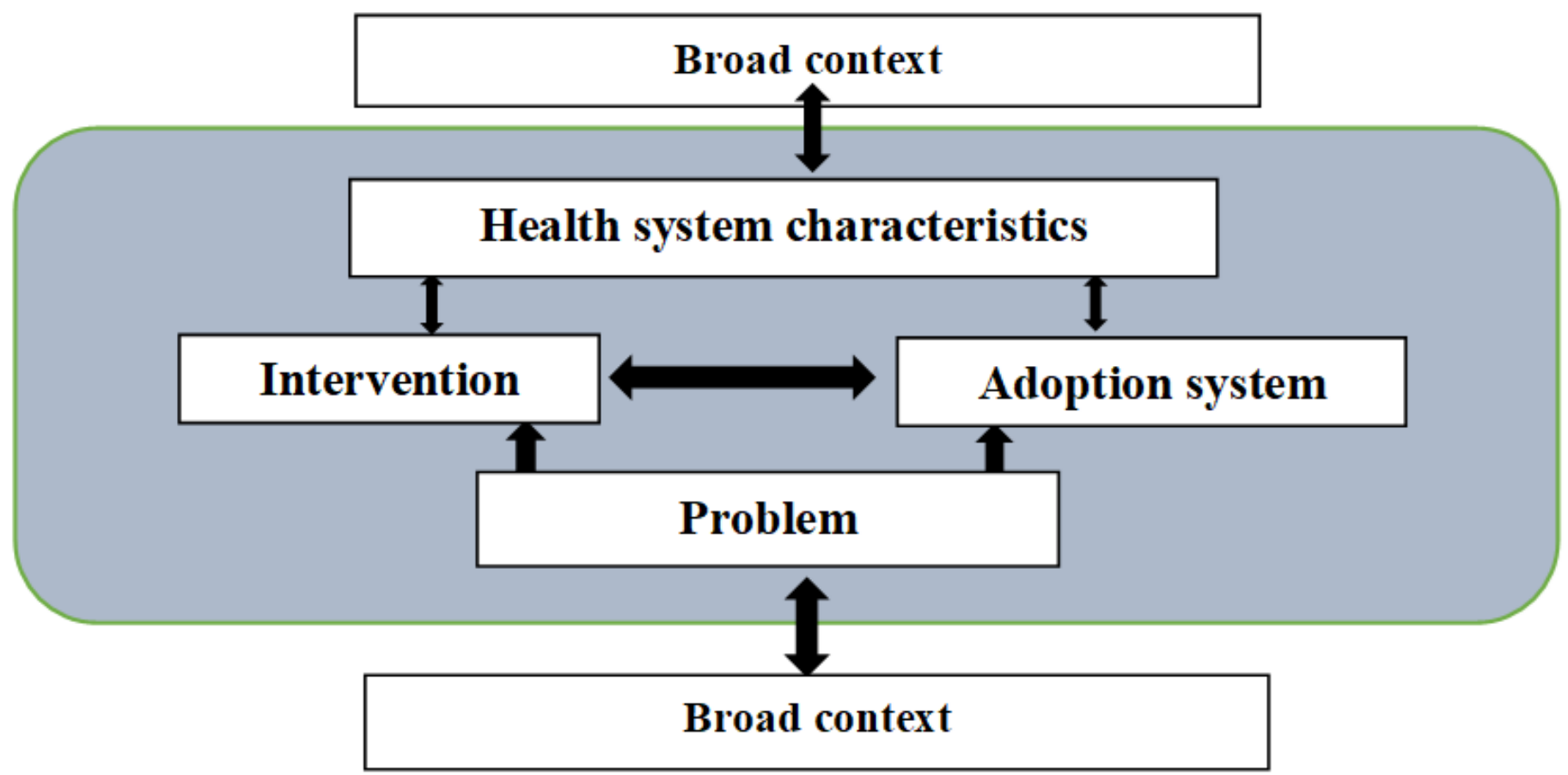

Figure 3 
Atun's integration framework

Page 23/23 